\title{
Unified description of Bjorken and Landau $1+1$ hydrodynamics
}

\author{
A.Bialas ${ }^{a}$, R.A.Janik ${ }^{a}$, and R.Peschanski ${ }^{b *}$ \\ ${ }^{a}$ M.Smoluchowski Institute of Physics, Jagellonian University, \\ Reymonta 4, 30-059 Krakow, Poland. \\ ${ }^{b} \mathrm{CEA} / \mathrm{DSM} / \mathrm{SPhT}$ Saclay Unité de Recherche associée au CNRS \\ CEA-Saclay, F-91191 Gif/Yvette Cedex, France.
}

\begin{abstract}
We propose a generalization of the Bjorken in-out Ansatz for fluid trajectories which, when applied to the $(1+1)$ hydrodynamic equations, generates a one-parameter family of analytic solutions interpolating between the boost-invariant Bjorken picture and the non boostinvariant one by Landau. This parameter characterises the propertime scale when the fluid velocities approach the in-out Ansatz. We discuss the resulting rapidity distribution of entropy for various freezeout conditions and compare it with the original Bjorken and Landau results.
\end{abstract}

\section{Introduction}

There is an accumulating evidence that hydrodynamics may be relevant for the description of the medium created in high-energy heavy ion collisions [1]. Indeed, experimental measurements such as the elliptic flow [2] shows the existence of a collective effect on the produced particles which can be described in terms of a motion of the fluid. More precisely, numerical simulations of the hydrodynamic equations describe quite well the distribution

\footnotetext{
*e-mails:bialas@th.if.uj.edu.pl,ufrjanik@th.if .uj.edu.pl,pesch@spht.saclay.cea.fr
} 
of low- $p_{\perp}$ particles [1], with an equation of state close to that of a "perfect fluid" with a rather low viscosity. This evidence is of course indirect, since it relies on assumptions about the initial and final stages of the evolution of the fluid. Thus some doubts can be cast either on the full thermalization of the medium, or on the possibility of accounting for some viscosity of the fluid [3]. Also, hydrodynamics are not expected to work for leading particles i.e. near the kinematic light-cone.

Given these objections, it is important to separate precisely the consequences of the hydrodynamic flow from those of the initial and final conditions. From that point of view, it seems tempting to discuss a simplified picture which can be qualitatively understood in physical terms. One such simplification, which we are going to follow in this paper, is the idea that the evolution of the system before freeze-out is dominated by the longitudinal motion $[4,5]$ and thus, in fact, the hydrodynamic transverse motion can be neglected or at least factorized out. Thus we shall consider the $(1+1)$ dimensional system.

On the theoretical grounds, there are also quite appealing features for applying hydrodynamic concepts to high-energy heavy-ion reactions. Such concepts have been already introduced some time ago and find a plausible realization nowadays. The fact that a rather dense medium is created in the first stage of the collision allows one to admit that the individual partonic or hadronic degrees of freedom are not relevant during the early evolution of the medium and justifies its treatment as a fluid. Moreover, the high quantum occupation numbers allow one to use a classical picture and to assume that the "pieces of fluid" may follow quasi-classical trajectories in space-time, expressed as an in-out cascade [6] with the straight-line trajectories starting at the origin, i.e.

$$
y=\eta
$$

where

$$
y=\frac{1}{2} \log \left(\frac{E+p}{E-p}\right) ; \eta=\frac{1}{2} \log \left(\frac{t+z}{t-z}\right)
$$

are respectively the rapidity and "space-time rapidity" of the piece of the fluid.

Note, for further use, that (1) can be rewritten in the form

$$
2 y=\log u^{+}-\log u^{-}=\log z^{+}-\log z^{-}
$$

where $u^{ \pm}=e^{ \pm y}$ are the light-cone components of the fluid (four-)velocity and $z_{ \pm}=t \pm z$ are the light-cone kinematical variables. 
Taking (1) as the starting point and using the perfect fluid hydrodynamics, Bjorken developped in his seminal paper [5] a suggestive (and very useful in many applications) physical picture of the central rapidity region of highly relativistic collisions of heavy ions. In this picture the condition (1) leads to a boost-invariant geometry of the expanding fluid and thus to the central plateau in the distribution of particles.

It is now experimentally established [7], however, that the central rapidity region of heavy ion collisions is better described by a Gaussian fit with a width proportional to $Y=\log s$, the total rapidity range of the secondaries. This finding has renewing interest [8] for the pioneering hydrodynamic description by Landau [4] where, indeed, a Gaussian-like distribution of the fluid was obtained. For the same reason, new families of $1+1$ relativistic hydrodynamic solutions have been recently proposed $[9,10]$.

In the present paper we propose to study a generalization of the formula (1) for the classical trajectory which (as we show in the following) interpolates naturally between the Landau and Bjorken pictures:

$$
2 y=\log u^{+}-\log u^{-}=\log f_{+}\left(z_{+}\right)-\log f_{-}\left(z_{-}\right)
$$

where $f_{ \pm}\left(z_{ \pm}\right)$are a priori arbitrary functions. They have to be determined from the hydrodynamic equations.

The hydrodynamic equations are rewritten in terms of light- cone variables in the next section. The consequences of the quasi-classical Ansatz (1) and of the generalized one (4) are dicussed in Section 3 where also the corresponding solutions of the hydrodynamical equations are derived. Various selections of the freeze-out conditions are discussed in Section 4. Our conclusions and comments are listed in the last section.

\section{Hydrodynamic equations in light-cone vari- ables}

We consider the perfect fluid for which the energy-momentum tensor is

$$
T^{\mu \nu}=(\epsilon+p) u^{\mu} u^{\nu}-p \eta^{\mu \nu}
$$

where $\epsilon$ is the energy density, $p$ is the pressure and $u^{\mu}$ is the 4 -velocity. We assume that the energy density and pressure are related by the equation of state:

$$
\epsilon=g p
$$


where $1 / \sqrt{g}$ is the sound velocity in the liquid. Using

$$
u^{ \pm} \equiv u^{0} \pm u^{1}=e^{ \pm y}
$$

and introducing

$$
z_{ \pm}=t \pm z=z^{0} \pm z^{1}=\tau e^{ \pm \eta} \rightarrow\left(\frac{\partial}{\partial z^{0}} \pm \frac{\partial}{\partial z^{1}}\right)=\frac{1}{2} \frac{\partial}{\partial z^{ \pm}} \equiv \frac{1}{2} \partial_{ \pm}
$$

where $\tau=\sqrt{z_{+} z_{-}}$is the proper time and $\eta$ is the spatial rapidity of the fluid, the hydrodynamic equations

$$
\partial_{\mu} T^{\mu \nu}=0
$$

take the form

$$
\partial_{ \pm} T^{01}+\frac{1}{2} \partial_{+}\left(T^{11} \pm T^{00}\right)-\frac{1}{2} \partial_{-}\left(T^{11} \mp T^{00}\right)=0 .
$$

Using now (5) and the equation of state (6) we deduce from this

$$
\begin{aligned}
& g \partial_{+}[\log p]=-\frac{(1+g)^{2}}{2} \partial_{+} y-\frac{g^{2}-1}{2} e^{-2 y} \partial_{-} y \\
& g \partial_{-}[\log p]=\frac{(1+g)^{2}}{2} \partial_{-} y+\frac{g^{2}-1}{2} e^{2 y} \partial_{+} y
\end{aligned}
$$

These are two equations for two unknowns which describe the state of the liquid: the pressure $p$ and the rapidity $y$. They should be expressed in terms of the positions $z_{+}, z_{-}$in the liquid. Other thermodynamic quantities can be obtained from the equation of state (6) and the standard thermodynamical identities:

$$
p+\epsilon=T s ; d \epsilon=T d s
$$

where we have assumed for simplicity vanishing chemical potential.

The result is

$$
\epsilon=g p=\epsilon_{0} T^{g+1} ; s=s_{0} T^{g} \rightarrow s \sim \epsilon^{g /(g+1)} .
$$

Note that (11) implies the consistency condition

$$
\partial_{+} \partial_{-} y=\frac{g^{2}-1}{4(1+g)^{2}}\left\{\partial_{-} \partial_{-}\left[e^{-2 y}\right]-\partial_{+} \partial_{+}\left[e^{+2 y}\right]\right\} .
$$




\section{Generalized in-out Ansatz}

\subsection{Bjorken's in-out Ansatz and boost-invariance}

The simplest possibility to describe the expansion of the fluid was suggested by Bjorken [5] who proposed to use the Ansatz (1) in the hydrodynamical context. Introducing (1) into (11) we obtain

$$
g \partial_{+}[\log p]=-\frac{1+g}{2 z_{+}} ; g \partial_{-}[\log p]=-\frac{g+1}{2 z_{-}}
$$

from which we deduce

$$
p=\epsilon g^{-1}=p_{0}\left(z_{+} z_{-}\right)^{-(g+1) / 2 g}=p_{0} \tau^{-(g+1) / g},
$$

where $p_{0}$ is a constant. Thus the system is boost-invariant: the pressure does not depend neither on $\eta$ nor on $y$. So are $\epsilon, s$ and $T$, given by (13).

\subsection{Beyond boost invariance}

The data on both nucleon-nucleon and nucleus-nucleus collisions (see, e.g. [7]) show that the produced system strongly violates boost invariance (except perhaps in a narrow region of small c.m. rapidities). It is thus necessary to go beyond (1). As already indicated in the Introduction, we propose to study -as a simplest generalization of (1)- the Ansatz (4). Introducing (4) into (14) we obtain

$$
f_{-} \partial_{-} \partial_{-}\left(f_{-}\right)=f_{+} \partial_{+} \partial_{+}\left(f_{+}\right)=A^{2} / 2
$$

where $A^{2}$ is a constant. Thus both $f_{+}$and $f_{-}$satisfy an identical equation:

$$
f f^{\prime \prime}=A^{2} / 2 \text {. }
$$

Note that $A=0$ implies $f^{\prime \prime}=0$ and thus we recover the condition (1). We conclude that $A$ describes the deviation of the system from the uniform Hubble-like expansion.

Eq. (18) can be solved multiplying by $f^{\prime}$ and dividing by $f$ :

$$
\left[\left(f^{\prime}\right)^{2}\right]^{\prime}=A^{2}[\log f]^{\prime} \rightarrow f^{\prime}=A \sqrt{\log (f / H)}
$$

where $H$ is an arbitrary constant. 
Eq. (19) can be solved in the standard manner. We obtain

$$
z-z_{0}=h \int_{F_{0}}^{F} \frac{d F^{\prime}}{\sqrt{\log \left(F^{\prime}\right)}}
$$

where we have introduced the notation

$$
F=f / H ; h=H / A .
$$

When (4) is introduced into (19) we obtain

$$
\begin{aligned}
& g \partial_{+}[\log p]=-\frac{(1+g)^{2}}{4} \frac{F_{+}^{\prime}}{F_{+}}+\frac{g^{2}-1}{4} \frac{F_{-}^{\prime}}{F_{+}} \\
& g \partial_{-}[\log p]=-\frac{(1+g)^{2}}{4} \frac{F_{-}^{\prime}}{F_{-}}+\frac{g^{2}-1}{4} \frac{F_{+}^{\prime}}{F_{-}} .
\end{aligned}
$$

From this we deduce

$$
\begin{aligned}
& g \log p=-\frac{(1+g)^{2}}{4} \log F_{+}+\frac{g^{2}-1}{4} F_{-}^{\prime} \int \frac{d z_{+}}{F_{+}}+\Delta_{-}\left(z_{-}\right) \\
& g \log p=-\frac{(1+g)^{2}}{4} \log F_{-}+\frac{g^{2}-1}{4} F_{+}^{\prime} \int \frac{d z_{-}}{F_{-}}+\Delta_{+}\left(z_{-}\right) .
\end{aligned}
$$

The integrals on the R.H.S. can be evaluated using (19). Indeed

$$
\int \frac{d z}{F}=\int \frac{d F}{F} \frac{1}{F^{\prime}}=h \int \frac{d \log F}{\sqrt{\log F}}=2 h \sqrt{\log F} .
$$

We thus obtain

$$
g \log p=-\frac{(1+g)^{2}}{4} \log \left(F_{+} F_{-}\right)+\frac{g^{2}-1}{2} \sqrt{\log \left(F_{+}\right) \log \left(F_{-}\right)} .
$$

where, for the two equations (23) to be consistent with each other, we had to take $\Delta_{ \pm}\left(z_{ \pm}\right)=-\frac{(1+g)^{2}}{4} \log F_{ \pm}$. This finally gives

$$
\begin{aligned}
& p\left(z_{+}, z_{-}\right)=p_{0} \exp \left\{-\frac{(1+g)^{2}}{4 g}\left[l_{+}^{2}+l_{-}^{2}\right]+\frac{g^{2}-1}{2 g} l_{+} l_{-}\right\} \\
& y\left(z_{+}, z_{-}\right)=\frac{1}{2}\left(l_{+}^{2}-l_{-}^{2}\right)
\end{aligned}
$$




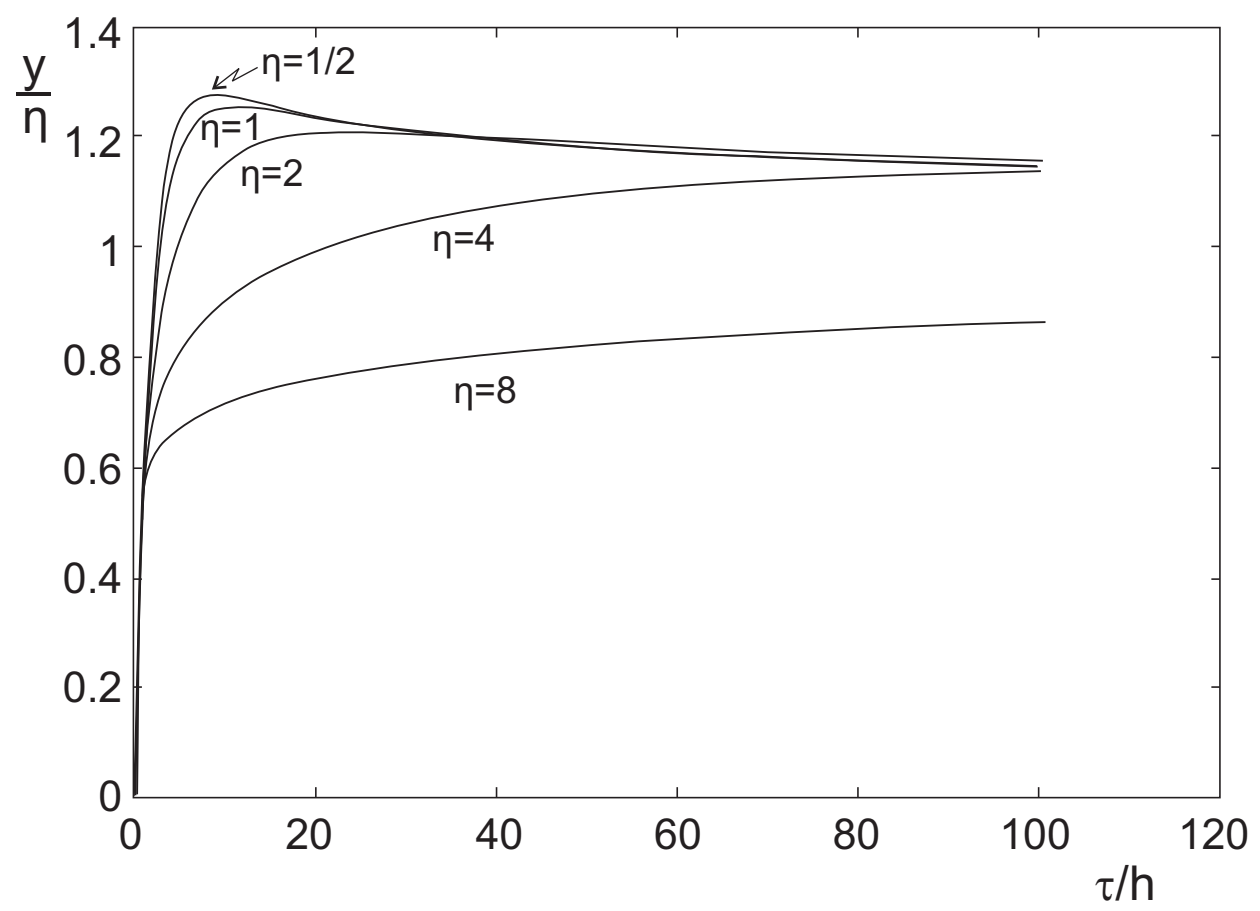

Figure 1: The ratio $y / \eta$ plotted versus proper-time $\tau / h$ for several values of space-time rapidity $\eta$. The boost-invariant picture corresponds to $y / \eta \equiv 1$. The "full-stopping" initial condition is $y / \eta=0$ at $\tau=0$. The asymptoticvalue is still 1 , but it is reached only at very large proper-times.

where, by definition

$$
l_{ \pm}\left(z_{ \pm}\right)=\sqrt{\log \left(F_{ \pm}\right)} .
$$

This completes the solution of hydrodynamic equations constrained by the generalized in-out cascade (4). One sees that the pressure depends on both $\tau$ and $\eta$. Thus the system is not boost-invariant but boost invariance is recovered in the limit $h \rightarrow 0, z_{ \pm}$fixed (see Eq. (20)). On the other hand, it can be remarked that the Landau asymptotic solution [4] can be recovered in the limit $h$ fixed, $z_{ \pm} \rightarrow \infty$.

Other thermodynamic parameters are obtained from (13), giving

$$
s \sim \epsilon^{g /(1+g)}=s_{0} \exp \left\{-\frac{1+g}{4}\left[l_{+}^{2}+l_{-}^{2}\right]+\frac{g-1}{2} l_{+} l_{-}\right\}=s_{0} \exp (-g \theta)
$$


where we have denoted

$$
\theta \equiv \log \left(T_{0} / T\right)=\frac{1+g}{4 g}\left[l_{+}^{2}+l_{-}^{2}\right]-\frac{g-1}{2 g} l_{+} l_{-}
$$

with $s_{0}$ and $T_{0}$ denoting the entropy and temperature at the beginning of the hydrodynamic evolution.

To illustrate the deviation of our solution (27) from the in-out BjorkenGottfried-Low Ansatz (1), we show in the Fig.1 the ratio $y / \eta$ vs $\tau / h$ (the proper time measured in units of $h$ ) for several (fixed) values of $\eta$. One sees that in the limit $\tau / h \rightarrow 0$ the rapidity $y$ vanishes. In this limit we have $(e . g$. for the region $y \geq 0$ )

$$
y \approx(\tau / 2 h)^{2} \sinh 2 \eta=(t / 2 h)^{2}-(z / 2 h)^{2} \leq \frac{1}{4}(t / h)^{2} .
$$

Thus for a fixed (small) $t / h$ the fluid starts at rest and acquires some velocity at later times, as in the Landau "full stopping" solution. At large times, $\tau \rightarrow \infty$, one obtains $y \approx \eta$, i.e. the in-out Ansatz (1) is approximately recovered. Thus our solution does indeed interpolate between the Landau and Bjorken hydrodynamics.

A last comment is in order. In all cases, the solution of the flow is also defined outside the kinematical light-cone. Indeed, there is some flow of energy entering the light-cone from outside. It could be physically interpreted as mimicking energy sources on the light-cone ("leading particle effect"). However, the relevance of hydrodynamical models near the light-cone is questionable.

\section{Entropy density at freeze-out}

The observable results of the model depend in an essential way on the assumed freeze-out surface. The point is that the densities $s$ and $\epsilon$ which enter the hydrodynamic equations are densities per unit volume in the rest frame of the fluid. But we are generally interested in the distribution of entropy $d S / d y$ and/or of energy $d E / d y$ densities per unit of rapidity, as these quantities are possible to measure. For given $s$ and $\epsilon, d S / d y$ and $d E / d y$ depend on the hypersurface at which the hydrodynamic evolution stops and the fluid changes into particles (freeze-out surface). To fix attention, in the following we discuss the entropy density. 


\subsection{General freeze-out surface}

The evaluation of the entropy density per unit of rapidity for a given freezeout surface can be performed in two steps.

First we evaluate the amount of entropy in an infinitesimal volume along the freeze-out surface:

$$
d S=s u^{\mu} d \sigma_{\mu}
$$

where $u^{\mu}$ is the 4 -velocity of the fluid and $d \sigma_{\mu}$ is the 4 -vector orthogonal to the surface satisfying

$$
d \sigma^{\mu} d \sigma_{\mu}=d z^{\mu} d z_{\mu}=d z_{+} d z_{-}
$$

Consider the (space-like) surface

$$
\Phi\left(z_{+}, z_{-}\right)=C
$$

where $C$ is a constant. Then

$$
\Phi_{+} d z_{+}+\Phi_{-} d z_{-}=0 ; \quad \Phi_{ \pm} \equiv \frac{\partial \Phi}{\partial z_{ \pm}} .
$$

It follows that the unit vector orthogonal to the surface is

$$
n_{+}=\frac{\Phi_{-}}{\sqrt{\Phi_{+} \Phi_{-}}} ; n_{-}=\frac{\Phi_{+}}{\sqrt{\Phi_{+} \Phi_{-}}} .
$$

The infinitesimal length along the surface is

$$
d \sigma=\sqrt{d z_{+} d z_{-}}=d z_{-} \sqrt{\Phi_{-} / \Phi_{+}}=d z_{+} \sqrt{\Phi_{+} / \Phi_{-}}
$$

Therefore

$$
u^{\mu} d \sigma_{\mu}=\left[u^{+} \Phi_{+}+u^{-} \Phi_{-}\right] \frac{d z_{-}}{2 \Phi_{+}}=\left[u^{+} \Phi_{+}+u^{-} \Phi_{-}\right] \frac{d z_{+}}{2 \Phi_{-}} .
$$

In the second step we express the infinitesimal volume along the freezeout surface in terms of the infinitesimal interval of rapidity. This can be done using the relation (20) which gives $z_{ \pm}$as a function of $F_{ \pm}=\exp \left(l_{ \pm}^{2}\right)$. We have

$$
d z_{ \pm}=\frac{d z_{ \pm}}{d F_{ \pm}} \frac{d F_{ \pm}}{d l_{ \pm}^{2}} d l_{ \pm}^{2}=h \frac{\exp \left(l_{ \pm}^{2}\right)}{l_{ \pm}} d l_{ \pm}^{2}
$$


Using this relation and (20), the R.H.S. of (38) can be expessed in terms of $l_{+}$and $l_{-}$.

This in turn can be expressed in terms of rapidity $y$ using (4) and the condition (34) which gives an additional relation between $z_{+}$and $z_{-}$and thus following (20) also between $l_{+}$and $l_{-}$. In particular, using the differential forms, we have

$$
d l_{ \pm}^{2}=\frac{ \pm 2 \Phi_{\mp} e^{l_{\mp}^{2}} / l_{\mp}}{\Phi_{+} \exp \left(l_{+}^{2}\right) / l_{+}+\Phi_{-} \exp \left(l_{-}^{2}\right) / l_{-}} d y \rightarrow d z_{ \pm}=\frac{ \pm 2 h \Phi_{\mp}}{\Phi_{+} l_{-} e^{-l_{-}^{2}}+\Phi_{-} l_{+} e^{-l_{+}^{2}}} d y
$$

and thus, finally

$$
u^{\mu} d \sigma_{\mu}=\frac{u^{+} \Phi_{+}+u^{-} \Phi_{-}}{\Phi_{+} l_{-} e^{-l_{-}^{2}}+\Phi_{-} l_{+} e^{-l_{+}^{2}}} d y=e^{\frac{1}{2}\left(l_{+}^{2}+l_{-}^{2}\right)} \frac{\Phi_{+} e^{y}+\Phi_{-} e^{-y}}{l_{-} \Phi_{+} e^{y}+l_{+} \Phi_{-} e^{-y}} .
$$

\subsection{Freeze-out at a fixed time}

In this section we take the surface at $t=$ const, for a first example. In the notation from the previous section we write

$$
\Phi\left(z_{+}, z_{-}\right)=t=\frac{1}{2}\left(z_{+}+z_{-}\right)=\text {const } ; \quad \Phi_{+}=\Phi_{-}=\frac{1}{2}
$$

Using (41) we thus have

$$
\begin{aligned}
d S=s u^{\mu} d \sigma_{\mu} & =\frac{s e^{\frac{1}{2}\left(l_{+}^{2}+l_{-}^{2}\right)}}{l_{+}+l_{-}+\left(l_{+}-l_{-}\right) \tanh y} d y= \\
& =\frac{s_{0} e^{-(g-1)\left(l_{+}-l_{-}\right)^{2} / 4}}{l_{+}+l_{-}+\left(l_{+}-l_{-}\right) \tanh y} d y .
\end{aligned}
$$

If, following Landau, we approximate both $l_{+}$and $l_{-}$by large constants, then for finite $y$ the difference $\left(l_{+}-l_{-}\right)$is small and we have

$$
d S \sim e^{-(g-1)\left(l_{+}-l_{-}\right)^{2} / 4} .
$$

For $g=3$ this formula is identical to the asymptotic result of Landau [4]. This can be seen when displaying the distribution $d S / d y$; In Fig.2, one shows $d S / d y$ with $t=$ const. (formula (43)) compared with the Landau approximation, formula (44), for different values of the parameter $h$, which, 


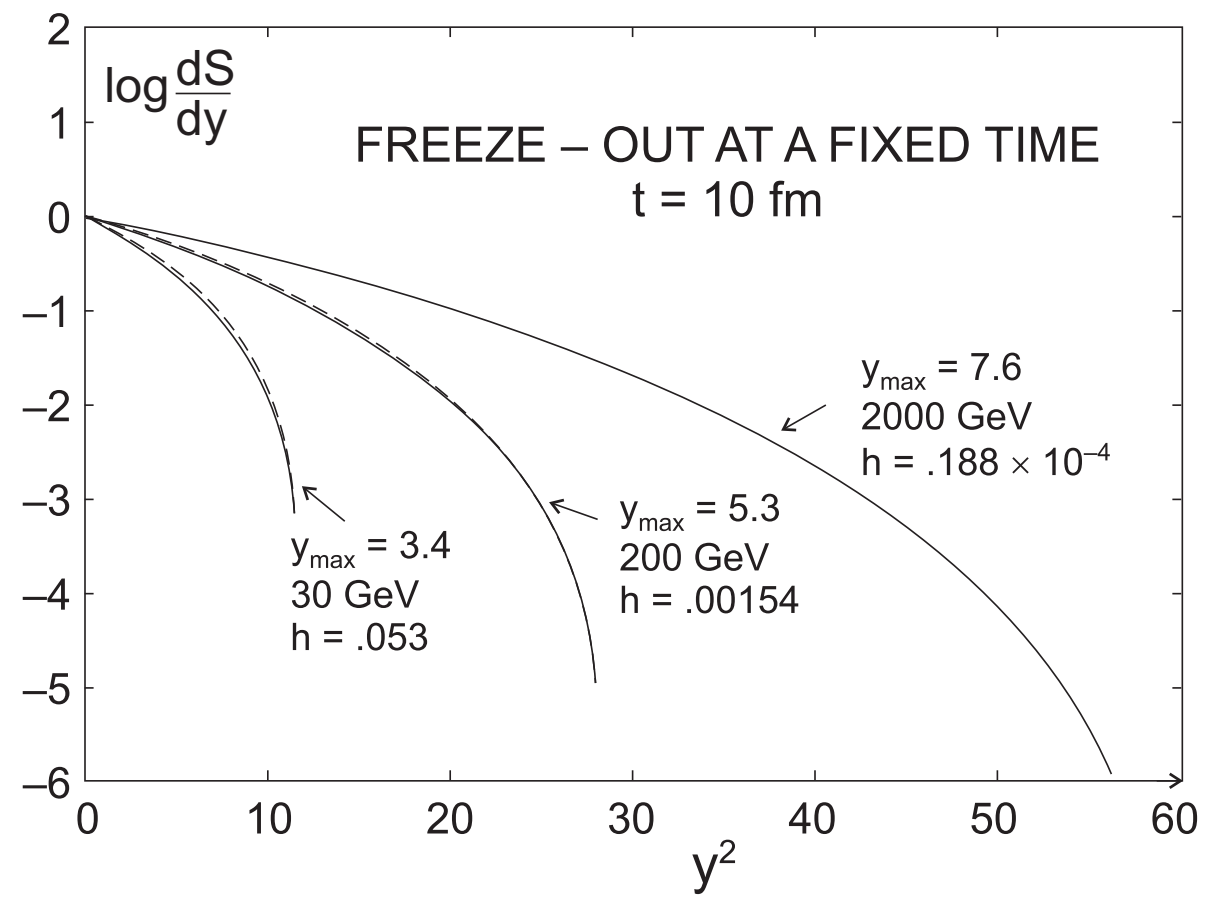

Figure 2: The curve $d S / d y$ with $t=$ const. (cf. formula (43)) compared with the Landau approximation (cf. (44), dashed lines). The kinematical end-points at $y_{\max }$ correspond to $z_{-}=0(y \geq 0)$.

by simple rescaling of the kinematic variables, correspond to different endpoints in rapidity.

One should keep in mind, however, that the relation $t=$ const. implies a different relation between $l_{ \pm}$and $y$ than the condition $\tau \sim$ const., which is the freeze-out condition considered ${ }^{1}$ by Landau [4]. As discussed in the next subsection, this leads to a rather different shape of the distribution $d S / d y$.

\subsection{Freeze-out at a fixed proper time}

To investigate the relation to the Landau solution and its comparison with the Bjorken one we consider the freeze-out at a fixed proper time.

\footnotetext{
${ }^{1}$ More precisely, Landau discusses the limitation of the $1+1$ dimensional motion by its transition to the $3+1$ dimensional hydrodynamical motion and shows that it appears at $\tau \sim$ const. playing the role of a freeze-out surface.
} 
Using the notation of Section 5.1 we have

$$
\begin{gathered}
\Phi\left(z_{+}, z_{-}\right)=z_{+} z_{-}=\tau^{2}=\text { const. } \\
z_{+} d z_{-}+z_{-} d z_{+}=0 ; \Phi_{ \pm}=z_{\mp}
\end{gathered}
$$

and thus

$$
d S=h e^{-(g-1)\left(l_{+}-l_{-}\right)^{2} / 4} \frac{e^{y} z_{-}+e^{-y} z_{+}}{l_{-} e^{y} z_{-}+l_{+} e^{-y} z_{+}} d y .
$$

This is a general formula. When supplemented by (4) and (20), it expresses $d S$ in terms $y$ and $\tau$.

When $h \rightarrow 0$ we can use the approximation (see the Appendix)

$$
z_{ \pm}=h \frac{F_{ \pm}}{l_{ \pm}}
$$

to obtain

$$
d S=h \frac{l_{+}+l_{-}}{2 l_{+} l_{-}} e^{-\frac{g-1}{4}\left(l_{+}-l_{-}\right)^{2}} d y .
$$

For $l_{ \pm} \rightarrow \infty$ and fixed $y$ one recovers the Landau result (44).

The result given by (47) is plotted in Fig.3 where $d S / d y$, is displayed for different values of the parameter $\mathrm{h}$ and compared with the approximate formula

$$
\frac{d S}{d y}=S_{0} e^{\sqrt{L^{2}-y^{2}}}
$$

obtained by Landau [4]. The parameter $L$ was adjusted to obtain the correct slope at $y=0$.

One observes some deviations from the perfect Gaussian which was considered in a simplified version [12] of the Landau model (and which agrees -if the multiplicity distribution is assumed proportional to the entropy- with the data [7]). Note that at fixed $\tau$ and $h \rightarrow 0$, the distribution becomes significantly flatter, going smoothly to the Bjorken limit at $h=0$.

\subsection{Freeze-out at a fixed temperature}

Instead of considering the freeze-out surface at the limit where the transverse motion becomes relevant (cf. [4]), a natural conjecture is to consider freeze-

out at a fixed temperature, i.e. when the temperature reaches the value where pions are expected to become liberated, e.g. $[1,11]$. 


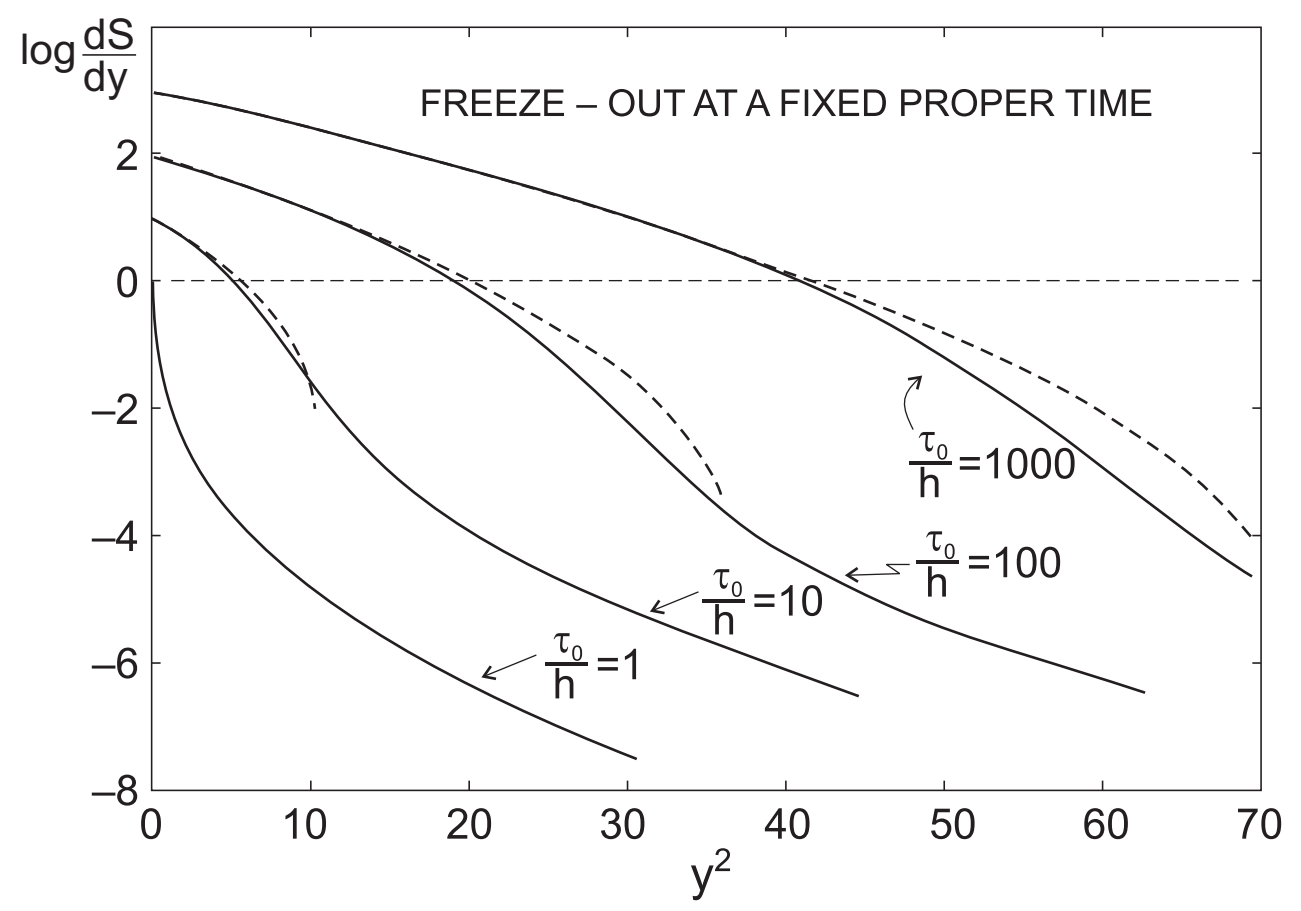

Figure 3: The curve $d S / d y$ with $\tau=$ const. for various values of $\tau / h$. Full lines: Eq.(49); Dashed lines: Eq.(50). Note that, for clarity, the curves were shifted by $\log _{10} \tau / h$.

Denoting the initial temperature by $T_{0}$ and the freeze-out temperature by $T_{F}$ we have

$$
\Phi\left(z_{+}, z_{-}\right) \equiv \frac{1+g}{4}\left[l_{+}^{2}+l_{-}^{2}\right]-\frac{g-1}{2} l_{+} l_{-}=g \log \left(T_{0} / T_{F}\right) \equiv g \theta=\text { const } .
$$

Hence

$$
\begin{aligned}
& \Phi_{+}=\frac{1}{2}\left[(g+1) l_{+}-(g-1) l_{-}\right] l_{+}^{\prime}=\frac{(g+1) l_{+}-(g-1) l_{-}}{4 h \exp \left(l_{+}^{2}\right)} \\
& \Phi_{-}=\frac{1}{2}\left[(g+1) l_{-}-(g-1) l_{+}\right] l_{-}^{\prime}=\frac{(g+1) l_{-}-(g-1) l_{+}}{4 h \exp \left(l_{-}^{2}\right)}
\end{aligned}
$$

where we have used the relation following from (20):

$$
l^{\prime}=\frac{d(\sqrt{\log F})}{d z}=\frac{F^{\prime}}{2 F \sqrt{\log F}}=\frac{1}{2 h F}=e^{-l^{2}} / 2 h .
$$


Therefore using (29) and (41) we have

$$
d S \sim e^{-(g-1)\left(l_{+}-l_{-}\right)^{2} / 4} \frac{l_{+}+l_{-}}{(g+1) l_{+} l_{-}-\frac{g-1}{2}\left(l_{+}^{2}+l_{-}^{2}\right)} .
$$

Now, the relations

$$
\frac{1+g}{4}\left[l_{+}^{2}+l_{-}^{2}\right]-\frac{g-1}{2} l_{+} l_{-}=g \theta ; \quad l_{+}^{2}-l_{-}^{2}=2 y
$$

imply

$$
l_{-}^{2}=\frac{g+1}{2} \theta-y+\frac{g-1}{2} \sqrt{\theta^{2}-y^{2} / g} ; \quad l_{+}^{2}=2 y+l_{-}^{2}
$$

giving

$$
\begin{aligned}
(g+1) l_{+} l_{-}-\frac{g-1}{2}\left(l_{+}^{2}+l_{-}^{2}\right) & =2 g \sqrt{\theta^{2}-y^{2} / g} \\
\frac{g-1}{4}\left(l_{+}-l_{-}\right)^{2} & =\frac{g-1}{2}\left[\theta-\sqrt{\theta^{2}-y^{2} / g}\right] \\
l_{+}+l_{-} & =\sqrt{2} y\left[\theta-\sqrt{\theta^{2}-y^{2} / g}\right]^{-1 / 2} .
\end{aligned}
$$

Thus we finally obtain

$$
d S \sim e^{\frac{g-1}{2}\left[\sqrt{\theta^{2}-y^{2} / g}-\theta\right]}\left(\theta-\sqrt{\theta^{2}-y^{2} / g}\right)^{-1 / 2} \frac{y d y}{\sqrt{\theta^{2}-y^{2} / g}} .
$$

One sees that this formula exibits a singularity ${ }^{2}$ by its transition to the $3+1$ dimensional hydrodynamical motion at $y^{2}=g \theta^{2}$ which is of course unphysical and reflects the singular initial conditions of our approach and the expected limitation of hydrodynamic models to the more central scattering region.

It is, however, interesting to observe that the hypersurface $T=$ const is only partly space-like. It becomes time-like at the rapidity determined from the condition $\Phi_{-}=0$, i.e., $(g+1) l_{-}=(g-1) l_{+}$, giving (c.f. (55))

$$
y_{m}=\frac{2 g}{g+1} \theta ; \quad l_{+}^{2}=(g+1) \theta .
$$

This is illustrated in Fig.4, where two profiles $\theta=$ const. are displayed.

\footnotetext{
${ }^{2}$ The singularity in $d S / d y$ does not come from a true singularity in the kinematical domain but is due to a maximum value reached by the rapidity $y$ as a function of $z_{-}$.
} 


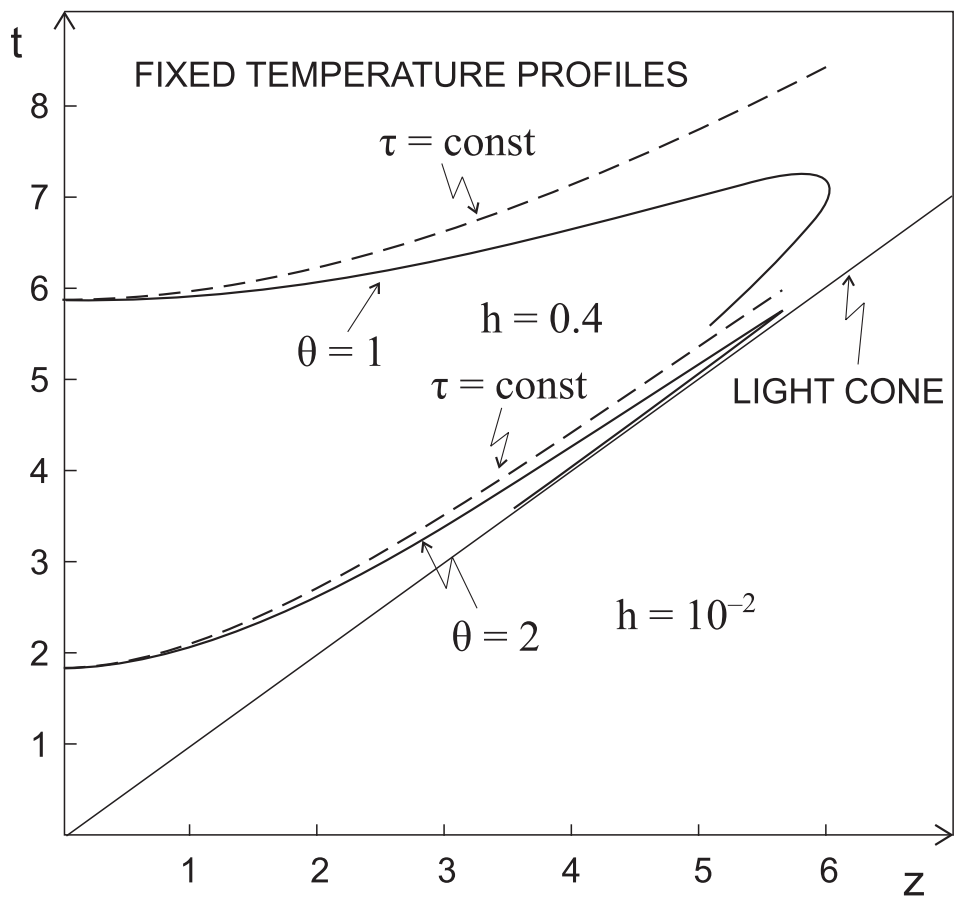

Figure 4: The profiles $\theta=$ const. for two values of $\theta=\log T_{0} / T$ (continuous lines). The comparison is made with fixed $\tau$ (dashed lines). Note that a different value of the parameter $\mathrm{h}$ has been chosen to obtain a comparable range in space-time.

The numerical estimates show that -for large enough $\theta(\theta \geq 2)$ - the effect of the singularity on the entropy distribution as a function of $y$ is not significant in the region $y \leq y_{m}$. This is shown in Fig. 5 where $d S / d y$ is plotted for several values of $\theta \geq 2$. One sees that all distributions are close to Gaussians. The slope, however, is rather small, certainly smaller than required by data, unless one considers a larger value of the parameter $g$, i.e. a smaller value of the speed of sound.

\section{Conclusions and outlook}

We have investigated longitudinal hydrodynamic expansion of a perfect fluid forming an infinitely thin layer at the initial time and satisfying the equation of state with an arbitrary sound velocity. We proposed a generalized in- 


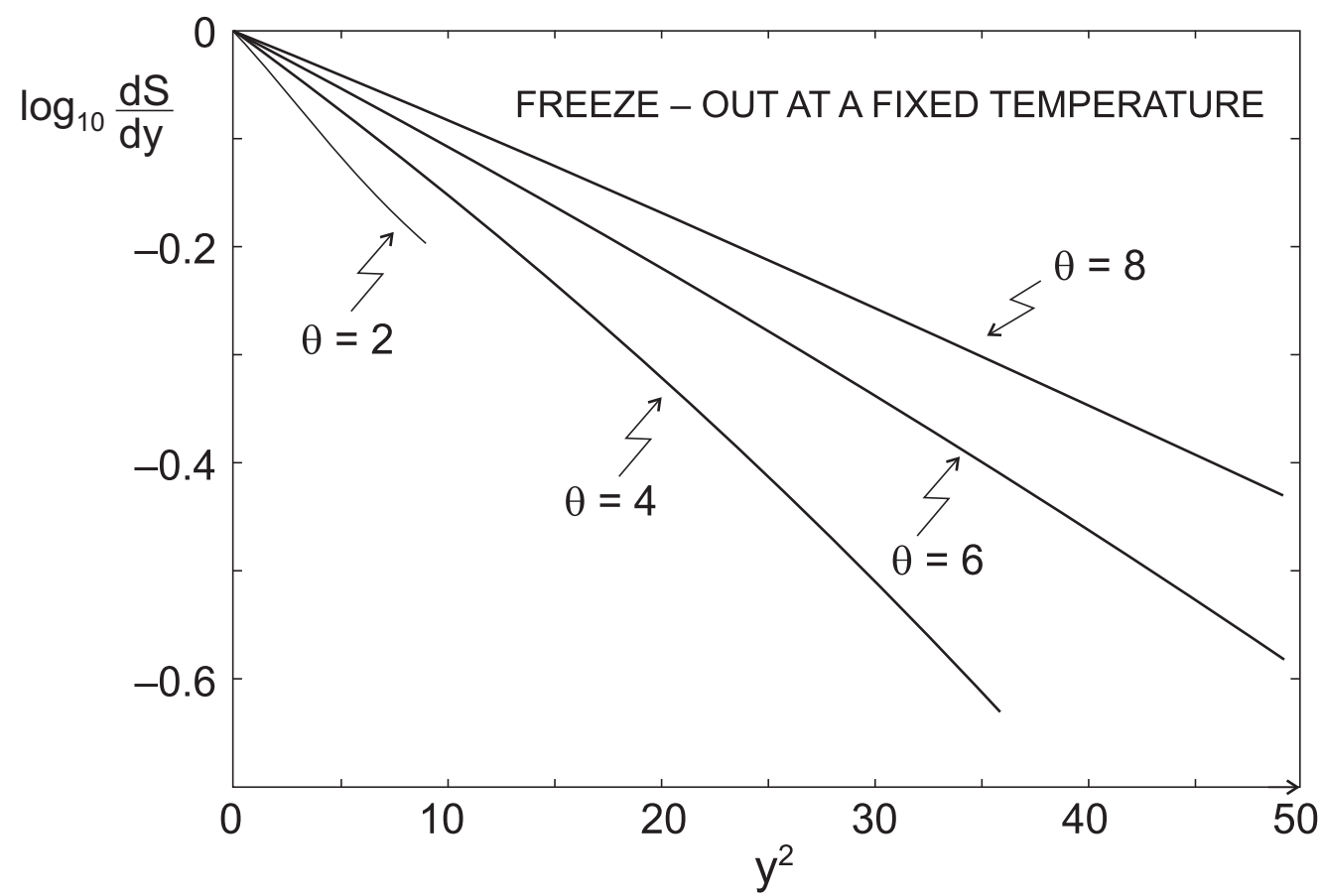

Figure 5: The curve $d S / d y$ with $\theta=$ const. for various values of $\theta, y<3 \theta / 2$.

out Ansatz which unifies the Landau and Bjorken approaches to relativistic hydrodynamics.

Our conclusions can be summarized as follows.

(i) When the Bjorken-Gottfried-Low in-out hypothesis (1) is assumed, the hydrodynamics implies that all thermodynamic properties of the fluid depend only on proper time, i.e. the system is boost-invariant.

(ii) We proposed a generalized in-out hypothesis, (4), and discussed its implications. It turns out that in this case the hydrodynamic equations can be solved in an analytic form, giving explicit formulae for the thermodynamic characteristics of the fluid in terms of their initial values and one free parameter $h$ defining a dynamical scale in configuration space.

(iii) The resulting entropy distribution in rapidity, $d S / d y$, was evaluated and shown to depend significantly on the assumed condition for the freezeout.

(a) For freeze-out at a fixed proper-time the density is close to (but with some deviation, particularly at small $\tau$, from) a Gaussian which is traditionally attributed to Landau solution. It tends smoothly to the Bjorken 
boost-invariant solution for $h \rightarrow 0$.

(b) For freeze-out at a fixed temperature the distribution is even closer to a Gaussian, if one restricts to the region where the freeze-out surface is space like and if the ratio $T_{0} / T_{F}$ is large enough.

(iv) It is worthwhile to note that the freedom in the choice of the value of the sound velicity may be helping in phenomenological applications of these results to data.

Compared to other recent $(1+1)$ hydrodynamical models $[9,10]$, our solution is mainly characterized by the smooth, one-parameter dependent transition between the Bjorken and Landau hydrodynamical models and by its analytic simplicity. It would be useful to study further the classification of all the solutions in a unified framework.

More generally, there is a clear need for an extension of our investigation to include more flexible initial conditions, relaxing the point-like character of the fluid at the beginning of the evolution. This, however, demands a more sophisticated analysis (e.g. an application of the general recipe of [13]) and goes beyond the scope of this paper.

On a theoretical ground, it would be interesting to have a physical interpretation of the generalized Ansatz (4), which appears as a mathematical harmonic property $\partial_{+} \partial_{-} y=0$ of the hydrodynamical flow. In particular, an extension to this case of the Gauge/Gravity correspondence applied in Ref.[14] to the Bjorken Boost-invariant flow, would be insightful. 


\section{Appendix. Solution of the equation (20)}

We rewrite (20) as

$$
(z-\zeta)=h \int_{F_{0}}^{F} \frac{d v}{\sqrt{\log v}}
$$

where $F=f / H$ and $h=H / A$.

Changing the variable of integration:

$$
\log v=u^{2} ; \quad 2 u d u=d v / v ; \quad d v / u=2 e^{u^{2}} d u
$$

we arrive at

$$
z-\zeta=2 h F \int_{\sqrt{\log F_{0}}}^{\sqrt{\log F}} e^{u^{2}-\log F} d u=2 \frac{H F}{A}\left[D(\sqrt{\log F})-D\left(\sqrt{\log F_{0}}\right)\right]
$$

This integral is the so called Dawson's integral: $D(x)=e^{-x^{2}} \int_{0}^{x} e^{u^{2}} d u$. For large $\sqrt{\log F}$ it approaches $(2 \sqrt{\log F})^{-1}$ and thus we obtain

$$
z-\zeta \approx \frac{H F}{A \sqrt{\log F}}
$$

The asymptotic expansion of $D(x)$ is

$$
D(x)=\frac{1}{2 x} \sum_{n=0}^{\infty} \frac{\Gamma(n+1 / 2)}{\Gamma(1 / 2)} \frac{1}{x^{2 n}}
$$

For small $x$ one can evaluate this integral effectively by the series expansion:

$$
D(x)=e^{-x^{2}} \int_{0}^{x} \sum_{n=0}^{\infty} \frac{x^{2} n}{n !}=x e^{-x^{2}} \sum_{n=0}^{\infty} \frac{x^{2 n}}{(2 n+1) n !}
$$

Thus we have in this case

$$
z-z_{0}=2 \frac{H}{A}\left[\sqrt{\log (F)} \sum_{n=0}^{\infty} \frac{(\log F)^{n}}{(2 n+1) n !}-\sqrt{\log \left(F_{0}\right)} \sum_{n=0}^{\infty} \frac{\left(\log F_{0}\right)^{n}}{(2 n+1) n !}\right]
$$




\section{Acknowledgements}

We thank Wojtek Florkowski, Jean-Yves Ollitrault and Kacper Zalewski for useful comments. This investigation was partly supported by the MEiN research grant 1 P03B 04529 (2005-2008) and by 6 Program of European Union "Marie Curie Transfer of Knowledge" Project: Correlations in Complex Systems "COCOS" MTKD-CT-2004-517186.

\section{References}

[1] See, for instance, T. Hirano, Acta Phys. Polon. B 36, 187 (2005) [arXiv:nucl-th/0410017];

P. Huovinen and P. V. Ruuskanen, Ann. Rev. Nucl. Part. Sci. 56, 163 (2006) [arXiv:nucl-th/0605008].

[2] J. Y. Ollitrault, Phys. Rev. D 46, 229 (1992); Nucl. Phys. A 638, 195 (1998) [arXiv:nucl-ex/9802005].

[3] R. S. Bhalerao, J. P. Blaizot, N. Borghini and J. Y. Ollitrault, Phys. Lett. B 627, 49 (2005) [arXiv:nucl-th/0508009].

H. J. Drescher, A. Dumitru, C. Gombeaud and J. Y. Ollitrault, "The centrality dependence of elliptic flow, the hydrodynamic limit, and the viscosity of hot QCD," arXiv:0704.3553 [nucl-th].

[4] L. D. Landau, "On the multiparticle production in high-energy collisions," Izv. Akad. Nauk Ser. Fiz. 17, 51 (1953) (in russian). [English translation: Collected Papers of L. D. Landau, edited by D. ter Haar (Gordon and Breach, New-York, 1968).]

[5] J. D. Bjorken, Phys. Rev. D 27, 140 (1983).

[6] J. D. Bjorken, "Hadron Final States In Deep Inelastic Processes," Lect. Notes Phys. 56, 93 (1976). Lectures given at the Int. Summer Inst. in Theoretical Physics, Current Induced Reactions, Hamburg, Germany, Sep 15-26, 1975. Also in Hamburg Theor.Inst. 1975:93 (QCD161:I83:1975).

K.Gottfried, Phys. Rev. Lett. 32957 (1974); F. E. Low and K. Gottfried, Phys. Rev. D 17, 2487 (1978). 
[7] See e.g. I. G. Bearden et al. [BRAHMS Collaboration], Phys. Rev. Lett. 94, 162301 (2005) [arXiv:nucl-ex/0403050], with a compilation of previous AGS and SPS Gaussian fits at lower energies.

[8] See, e.g. P. Steinberg, Acta Phys. Hung. A 24, 51 (2005) [arXiv:nucl$\mathrm{ex} / 0405022]$.

[9] T. Csorgo, M. I. Nagy and M. Csanad, "A New Family of Simple Solutions of Perfect Fluid Hydrodynamics," arXiv:nucl-th/0605070.

[10] S. Pratt, Phys. Rev. C 75, 024907 (2007) [arXiv:nucl-th/0612010].

[11] F. Cooper, G. Frye and E. Schonberg, Phys. Rev. D 11, 192 (1975).

[12] P. Carruthers and M. Doung-van,, Phys. Lett. B 41, 597 (1972); Phys. Rev. D 8 (1973) 859.

[13] I.M.Khalatnikov, Zh.Eksp. Teor. Fiz. 26529 (1954) (in Russian).

[14] R. A. Janik and R. Peschanski, Phys. Rev. D 73, 045013 (2006) [arXiv:hep-th/0512162]. 